\title{
Two become one
}

\author{
Developmental biologists and cell biologists have long ploughed their \\ separate furrows. But now these two disciplines are coming together in \\ unexpected and exciting ways, says Helen Pearson.
}

$\mathrm{K}$ athryn Anderson wasn't looking to embark on an affair with cell biology developmental biologist at the SloanKettering Institute in New York, was studying 'open-brain' mice - which have a fatal genetic mutation that prevents their brain from folding correctly during embryonic development - when her research took a surprising turn.

She had expected the gene involved to be one of the usual suspects implicated in severe developmental abnormalities - those encoding proteins that regulate the expression of other genes, or that are involved in key cellular signalling pathways known to direct embryological development. Instead, she found that the gene produced a protein that carries materials around the cell ${ }^{1}$. Such 'housekeeping' proteins had long been ignored by developmental biologists deemed of interest only to researchers fascinated with the detailed mechanics of a cell.

Anderson is just one of many developmental biologists who are now realizing that apparently mundane aspects of cells' internal workings can help to illuminate some of the mysteries of development. "For whatever reason, developmental geneticists have encountered basic cell biology in the past few years," she says. Cell biologists are likewise coming to recognize that their obsession with the mechanics of processes such as cell division, and the transport of proteins around the cell, can contribute to the study of development. Helping to broker this marriage between fields are techniques of video microscopy, which use fluorescent markers to film proteins moving around in cells and tissues (see 'Lights, camera, action!', overleaf).

For the past two decades, developmental geneticists have been busy identifying 'big hitting' developmental genes. These encode the proteins involved in signalling pathways that direct the organization of animals' tissues and organs. But knowing that the brain, for instance, is directed to grow in the way it does because gene $A$ turns on gene $B$ turns on gene $\mathrm{C}$, and so on, still leaves much to be learned about the developmental processes that underlie the complexity of the finished organ.

"Over the past five years there has been a shift away from the formal logic of genetic pathways," says Stephen Cohen, who studies the development of wings in the fruitfly Drosophila at the European Molecular Biology Laboratory in Heidelberg. This has seen many developmental biologists migrate into the territory staked out by their counterparts in cell biology. In retrospect, says Cohen, it was an obvious move. Developmental biologists consider how tissues and organs form — but these are simply populations of cells working together. So gaining a complete picture of how organisms are built will require an understanding of cells' internal workings.

\section{Steeped in gradients}

One area of developmental biology being revitalized by this new wave of thinking is the study of morphogens. These are secreted proteins, many of which trigger the signalling pathways that developmental geneticists have been teasing apart. According to the textbooks, morphogens work their magic by diffusing from a source to form a high-tolow gradient. Cells lying in the path of the diffusing morphogen sense its concentration in order to work out where they sit in a developing organ or tissue and, accordingly, what genes they need to switch on.

But this view of cells passively receiving cues from a protein diffusing around them was challenged two years ago by Tom Kornberg and FelipeAndrés Ramírez-Weber of the University of California, San Francisco. Far from just lying down and taking

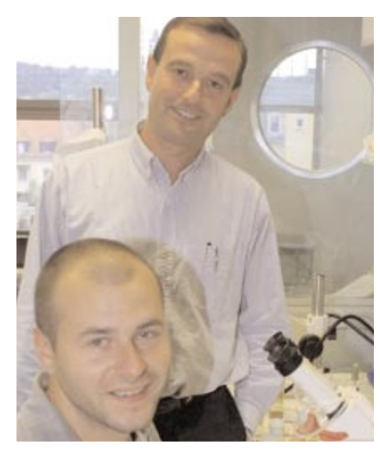

Marcos González-Gaitán (above, rear) has imaged the formation of a morphogen gradient (green) in fruitflies.

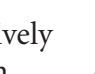

\section{rate}
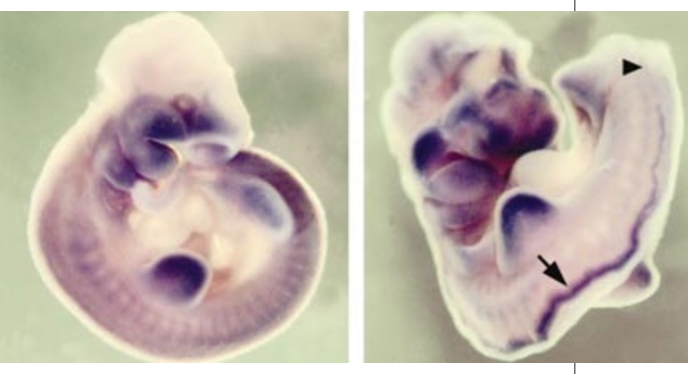

The mutated gene found in open-brain mouse embryos (right) offers a surprising link between cell biology and development.

their instructions from morphogens, it seems that cells often play an active role in determining how the gradient forms.

Kornberg and Ramírez-Weber were looking at the disc of developing tissue that ultimately gives rise to a fruitfly wing. They labelled cells at the edge of the disc with green fluorescent protein (GFP), a commonly used fluorescent marker. This revealed long thread-like fingers, which the researchers dubbed 'cytonemes', extending from these cells towards 
disc's dark, unlabelled centre, where a morphogen called Decapentaplegic (Dpp) is produced $^{2}$. Cells actively seek out morphogens by extending these protrusions, Kornberg and Ramírez-Weber proposed — although they have yet to prove that morphogens are actively channelled along the cytonemes.

\section{Disc drive}

Other researchers have labelled key fruitfly morphogens such as Hedgehog, Wingless and Dpp with fluorescent markers and watched the concentration gradients form. "It has changed the way I look at things," says Marcos González-Gaitán of the newly opened Max Planck Institute of Molecular Cell Biology and Genetics in Dresden (see this issue's NatureJobs, pages 4-5), which is concentrating on the interface between cell biology and development.

By fusing Dpp to GFP, González-Gaitán and his colleagues were able to watch a gradient form in the fruitfly wing disc. Some of the Dpp could clearly be seen in small round dots inside cells that the researchers think are endosomes - membrane-bound vesicles that form when substances are actively taken up by cells. And in discs containing patches of mutant cells unable to form endosomes, the flow of the morphogen through the tissue was impeded ${ }^{3}$."Instead of going between cells it's going through them," says González-Gaitán.

This means that the uptake and subsequent release of morphogens by individual cells may help to determine how quickly and how far they move through a tissue, and so determine the 'shape' of the concentration gradient that

forms.

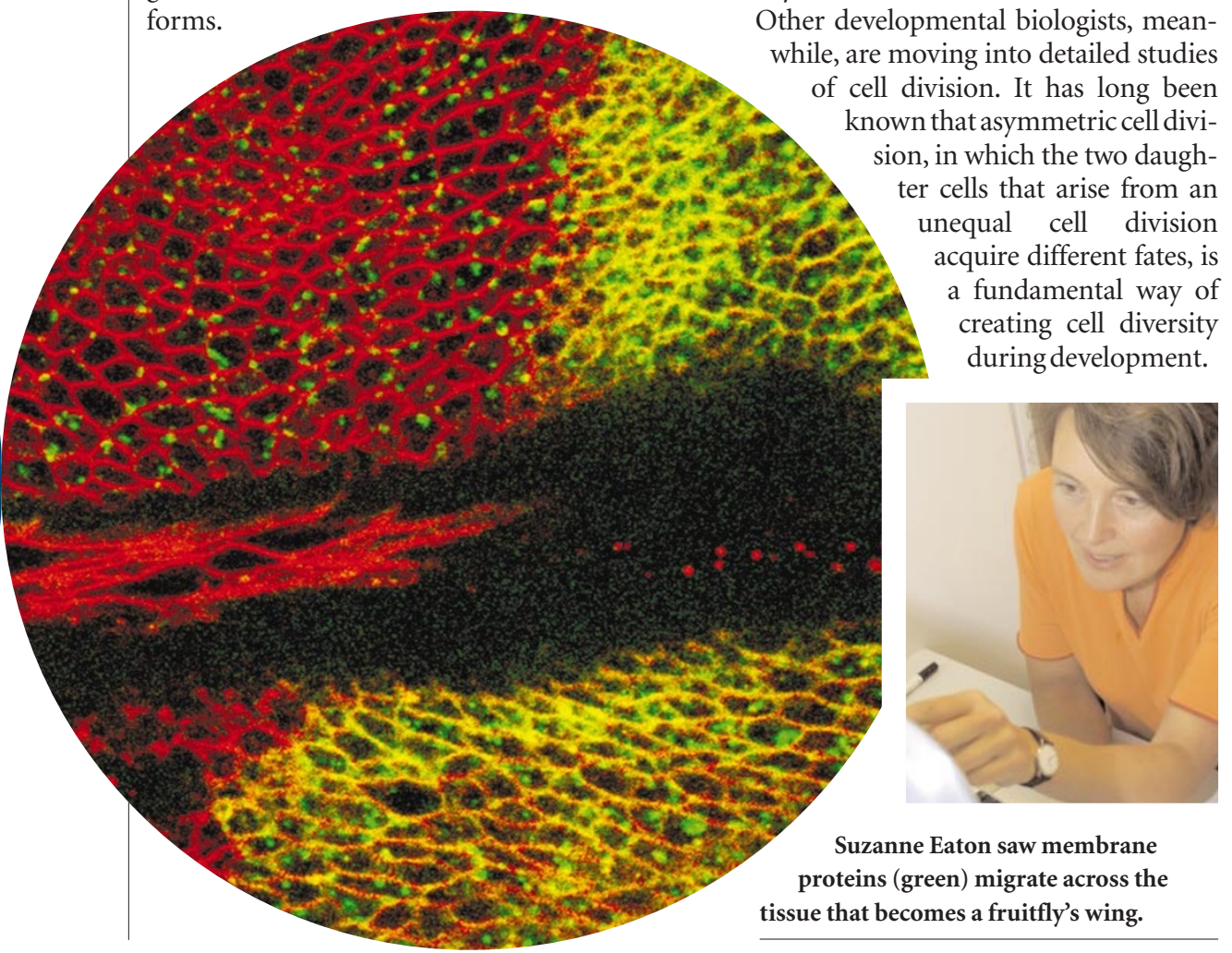

A related cellular process - the speed at which morphogens are broken down after being taken up into endosomes - may also be key in shaping morphogen concentration gradients. Wingless is a morphogen that normally accumulates in a series of graded stripes across the body of a developing fruitfly embryo, and is involved in specifying the insect's segmented body plan. But when Jean-Paul Vincent of the National Institute for Medical Research in London blocked the protein's breakdown using a drug called chloroquine, the concentration gradient changed, with the Wingless stripes extending into areas that do not normally contain the morphogen ${ }^{4}$.

This month, Suzanne Eaton, also at the Dresden Max Planck Institute, published another observation that may help to explain morphogen trafficking. She was studying how proteins are sorted to one side or the other of the fruitfly wing disc. She tagged GFP to a membrane protein expressed on only one side, but she noticed tiny glowing dots inside cells on the other, unlabelled side. These dots seem to form as tiny membrane-bound packages within larger endosomes. Eaton calls them 'argosomes', and speculates that they are released when endosomes fuse with cells' outer membranes - allowing morphogens to be passed from one cell to another.

The mechanisms being touted to explain the formation of morphogen gradients are not mutually exclusive, and it is possible that different processes come into play for different morphogens, and for the movement of the proteins over different distances. "Everyone would like to be the one who figures it out," says Eaton.

Other developmental biologists, meanefer cell division. It has long been in, in which the two daughcells that arise from an cquire different fates, is fundamental way of creating cell diversity

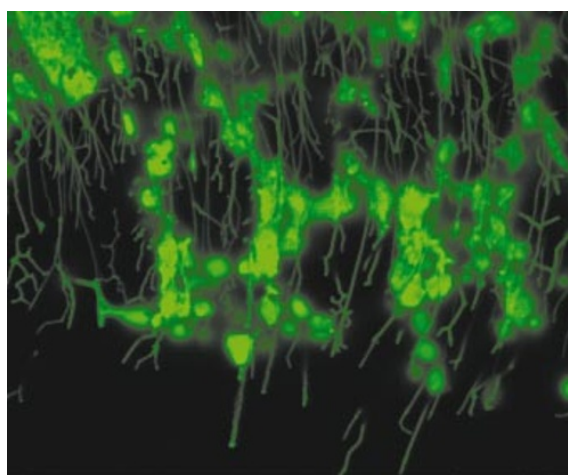

Green-fingered: are the thread-like cytonemes that reach out from cells searching for morphogens?

For this to achieve the desired results, the cells must be 'polarized' - they must have a 'top' and a 'bottom' in relation to the developing body plan. The fate of the daughter cells depends on the distribution of key proteins called determinants. Over the past few years, researchers have shown that asymmetric cell division depends both on the localization of these proteins within the parent cell before division, and on the orientation of a structure called the mitotic spindle, along which replicated chromosomes move to opposite ends of the cell before it divides. The spindle determines the plane of cell division, and its positioning influences the relative sizes of the resulting daughter cells, and the quantities of determinants that each receives.

\section{Determined efforts}

Back in 1994, a protein determinant called Numb was shown to accumulate in a crescent at one end of cells in fruitfly embryos that give rise to neurons ${ }^{6}$. Since then, researchers have identified a host of other determinants that also become localized to one or the other end of asymmetrically dividing cells, and they are busy investigating how this localization occurs ${ }^{7}$.

Meanwhile, other groups are investigating the orientation of the mitotic spindle. Andrea Brand and her team at the University of Cambridge, for instance, have revealed that a sudden shift in the spindle's axis is crucial to the development of the fruitfly nervous system. The cells that give rise to neurons develop from a simple layer of cells in which Numb, and another determinant called Prospero, are concentrated at the base of each cell. In the divisions that form this layer, the plane of cell division runs from top to bottom of the parent cell — which means that equal quantities of the determinants end up in the daughter cells. But some cells, called neuroblasts, detach from the layer and divide asymmetrically into a large neural stem cell and a smaller cell that inherits Prospero and Numb and subsequently divides to give rise to two neurons.

Brand's team showed that the change between symmetric and asymmetric division involves a simple switch of spindle 
